\title{
ANAESTHESIA AND TRAUMATIC DIAPHRAGMATIC HERNIA
}

\author{
T.R. LoBB, M.D. AND G.R. ButLin, M.D., C.R.C.P. (c) ${ }^{\circ}$
}

BLUNT TRAUMa to the chest or abdomen can result in a torn diaphragm with prolapse of abdominal contents into the thoracic cavity. ${ }^{3-3}$ Motor vehicle accidents cause the vast majority of these mishaps, although falls and crushing injuries may also be responsible. The incidence at Victoria Hospital, London, Ontario, approximated 1 in every 4,250 cases of motor vehicle trauma admitted over a five-year period (1966-1971). The mortality was approximately 25 per cent. Since reports of the anaesthetic experience in this condition are almost non-existent, we feel it appropriate, in view of the rising rate of injuries sustained in automobile accidents, to discuss the clinical presentation and present the plan of management generally practised at this centre.

Miller and Howie ${ }^{2}$ suggest that three factors are involved in the causation of traumatic diaphragmatic hernia:

1. Abdomino-thoracic pressure gradients created by sudden compression, and augmented by the "gasp" reflex.

2. Distortion of the diaphragm by chest compression resulting in shearing forces which act to produce a tear.

3. Congenital weakness of the diaphragm.

With the protection afforded to the right leaf of the diaphragm by the liver, about 95 per cent of these herniae occur through the left diaphragm. Stomach, spleen, transverse colon and small bowel are the most common organs to become incarcerated in the pleural cavity, causing pulmonary compression and varying degrees of mediastinal shift. The resultant cardiopulmonary embarrassment may be further augmented by pulmonary contusion, pneumothorax or acute blood loss from associated injuries such as fractures and lacerations. Laceration of the spleen is particularly common. Rarely, the hermiation may enter the pericardial sac alone, resulting in tamponade.

The symptoms, when prominent, are similar to those of a tension pneumothorax and consist of chest pain, dyspnoea and possibly cyanosis in conjunction with decreased air entry over the involved side. The presence of bowel sounds in the chest is not a reliable sign, since they are usually decreased or absent. A plain chest X-ray should either establish the diagnosis by showing gas-filled loops of bowel in the chest, or suggest it by the deceptive appearance of a unilaterally elevated diaphragm. A barium swallow is not usually helpful in the acute phase, and it carries the added risk of regurgitation and aspiration.

Once the diagnosis is established, immediate surgical correction is warranted; however, on occasion the diagnosis may not become apparent until years after the initial injury, and only then because of vague upper abdominal complaints. ${ }^{4}$

"Department of Anaesthesia, University of Western Ontario and Victoria Hospital, London, Ontario, Canada. 


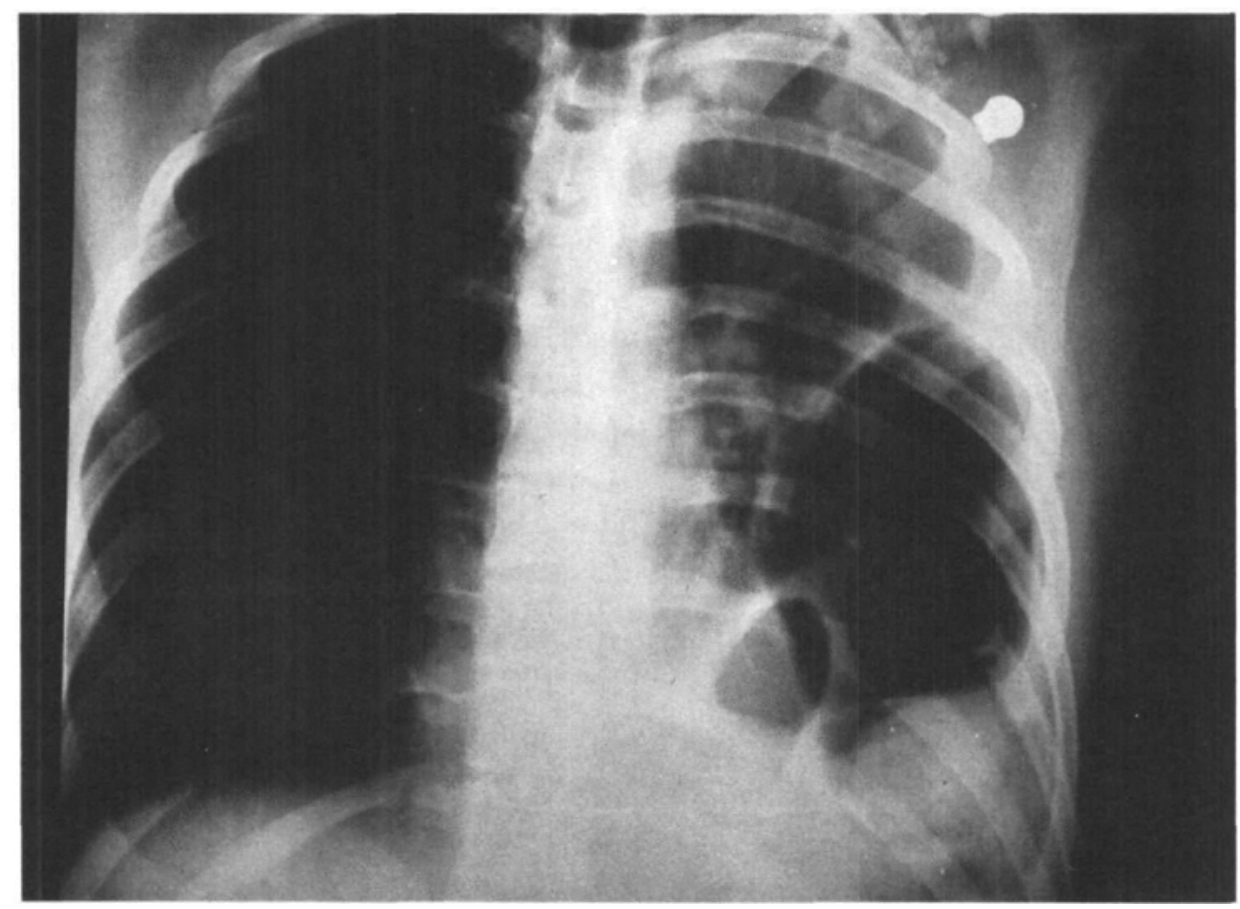

Figure 1. Case 1 (admission X-ray).

\section{Case Presentations}

Reflecting a rising highway casualty toll, five cases presented themselves in our city, a referral center, during the year of 1972. Three cases of the authors' experience occurred within three weeks of each other, and are now presented.

\section{Case 1}

A 17-year-old male was referred with the diagnosis of left diaphragmatic hernia, fractured pelvis and femur, and possible abdominal injuries after a motor vehicle accident. On arrival he was dyspnoeic with decreased air entry over the left chest in association with a rigid and silent abdomen. The left thigh was tense and swollen. His blood pressure was $96 / 70 \mathrm{~mm} \mathrm{Hg}$, the pulse rate $100 / \mathrm{min}$ and respirations $28 / \mathrm{min}$. $\mathrm{X}$-rays of the chest (Figure 1) showed a left haemothorax with a gas-filled loop, as well as a fractured pelvis and femur.

Because of marked respiratory distress, tracheal intubation was performed with the patient awake and ventilatory assistance given with an oxygen driven Bird Mark VII ventilator using a low pressure setting. Subsequently anaesthesia was induced with halothane and low tidal volume ventilation. At thoracotomy, the left hemithorax was found to contain one litre of blood along with the stomach, colon, jejunum and spleen, protruding through a 10 -centimetre tear in the diaphragm. The tear was repaired, and the incision extended into the abdomen for splenectomy and repair of a ruptured urinary bladder. Finally, K-wire fixation was applied to the leg, and the patient removed to the Intensive Care Unit having received six units of whole blood.

The tracheal tube was left in place for 24 hours of mechanical ventilation, and was then removed. The trachea was re-intubated briefly the following day for suctioning and re-inflation of an atelactatic lung segment. On the third post-operative day he became quite dyspnoeic with moderate hypoxaemia as revealed by blood gas determination. Fat embolization was suggested by a chest X-ray (Figure 2), and was treated 


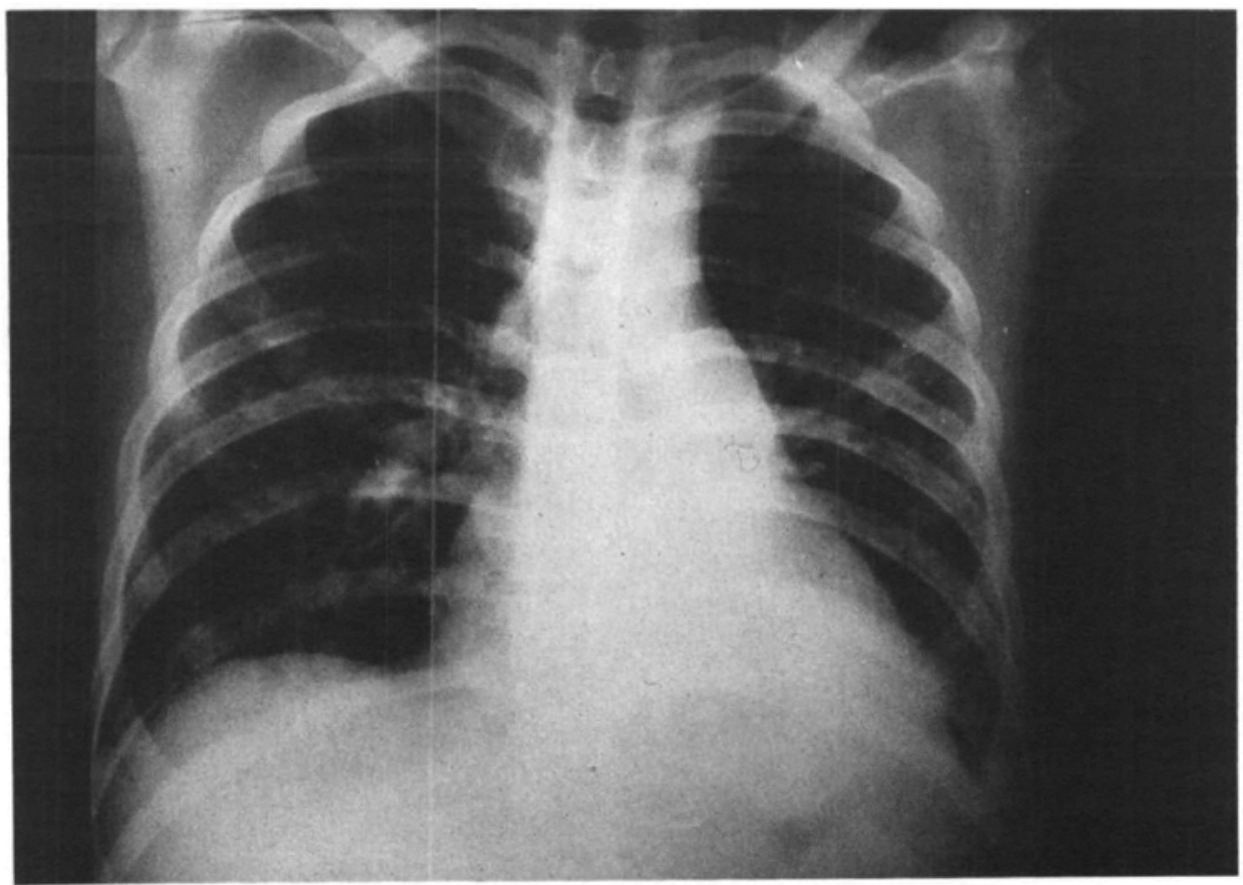

FIGURE 2. Case 1 (X-ray suggestive of fat embolization on third post-operative day).

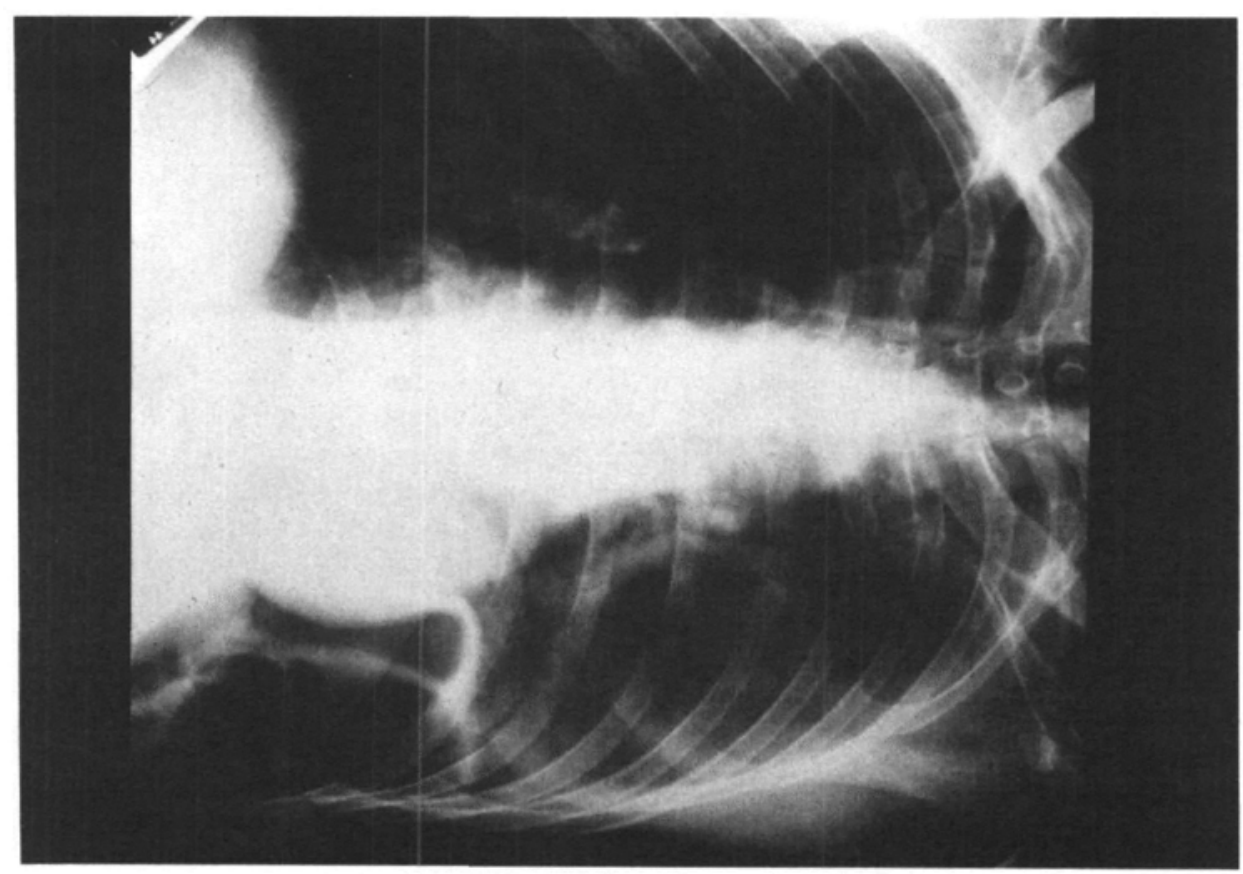

Figure 3. Case 2 (admission X-ray). 
conservatively with oxygen supplementation by mask. His chest cleared in three days. Blood gases breathing room air were near normal two weeks later. Bladder problems associated with the initial trauma delayed discharge until two months after admission.

Case 2

A 44-year-old male accident victim was transferred from an outlying hospital with a diagnosis of basal skull fracture and contused left lung. On arrival he was confused and restless, with bloody cerebrospinal fluid oozing from both ears. He was dyspnoeic and had decreased air entry in the left chest. The abdomen was tender, with decreased bowel sounds. A four-quadrant tap for blood had been negative. Blood pressure at that time was $138 / 90 \mathrm{~mm} \mathrm{Hg}$, the pulse rate $108 / \mathrm{min}$ (later rising to $140 / \mathrm{min}$ ), and respirations $28 / \mathrm{min}$. X-ray films showed a contused left lung with rib fractures and a gas-filled loop of bowel above the left diaphragm (Figure 3).

This patient was taken immediately to the operating room where major intravenous routes were established, including cannulation of an external jugular vein for central venous pressure monitoring. A nasogastric tube was inserted and suction applied. Following pre-oxygenation and intravenous Innovar ${ }^{\circledR}(1.5 \mathrm{cc})$, tracheal intubation was performed with the patient awake. Following a sleep dose of thiopentone, analgesia was maintained with nitrous oxide, oxygen and titrated increments of Innovar. Spontaneous respiration was allowed to continue until the surgeon was about to make his abdominal incision, at which time d-tubocurarine was added, and the ventilation was gently and gradually controlled. The spleen, stomach and large bowel were then quickly withdrawn into the abdominal cavity through a large tear in the posterior part of the left diaphragm. After splenectomy and following the insertion of two chest drainage tubes because of an air leak, he was taken to the Intensive Care Unit where mechanical ventilation was given overnight. The trachea was extubated the following morning. Persistent confusion and restlessness, as a result of his head injury, was subsequently compounded by hypoxaemia due to atelectasis, effusions and consolidation as shown on chest $\mathrm{X}$-rays. Treatment consisted of oxygen therapy in conjunction with antibiotics, humidification, intermittent positive pressure nebulizations and physiotherapy. His mental state improved, as did his pulmonary complications, and he was discharged home 32 days after admission.

\section{Case 3}

A 54-year-old male presented in moderate respiratory distress after a car accident. Besides superficial injuries, his left chest wall was tender in the presence of subcutaneous emphysema. There was decreased air entry over the left chest. The abdomen was soft. There was no tenderness and bowel sounds were normal. Vital signs showed a blood pressure of $140 / 70 \mathrm{~mm} \mathrm{Hg}$, a pulse rate of $80 / \mathrm{min}$ and respirations $20 / \mathrm{min}$. Blood gas determinations showed moderate hypoxaemia with normal acid-base balance. Initial X-rays (Figure 4) demonstrated a left haemo-pneumothorax with "elevated left diaphragm and a gas-distended stomach beneath," fractured ribs and tissue emphysema. There were also fractures of the left femur, tibia and fibula.

After the insertion of a chest drainage tube connected to an underwater seal, he was taken to the operating room for suturing and fixation of his fractures. He was given a general anaesthetic through a tracheal tube, using mechanical ventilation. The ventilation was continued post-operatively in the Intensive Care Unit for management of the multiple chest injuries. Poor pulmonary compliance made ventilatory control difficult. Subsequent weaning from the respirator was difficult and it was only after seven days that extubation was finally possible.

The possibility of diaphragmatic hernia was considered on review of his admission chest X-ray (Figure 4); however, radiography following instillation of barium contrast medium through a nasogastric tube (Figure 5) was inconclusive. It was only on repeat films five days later that a gas-filled loop was demonstrated in the left thorax above the retained barium. At operation a diaphragmatic hernia was repaired through a left thoracotomy, and the lungs were ventilated in the Intensive Care Unit for another 48 


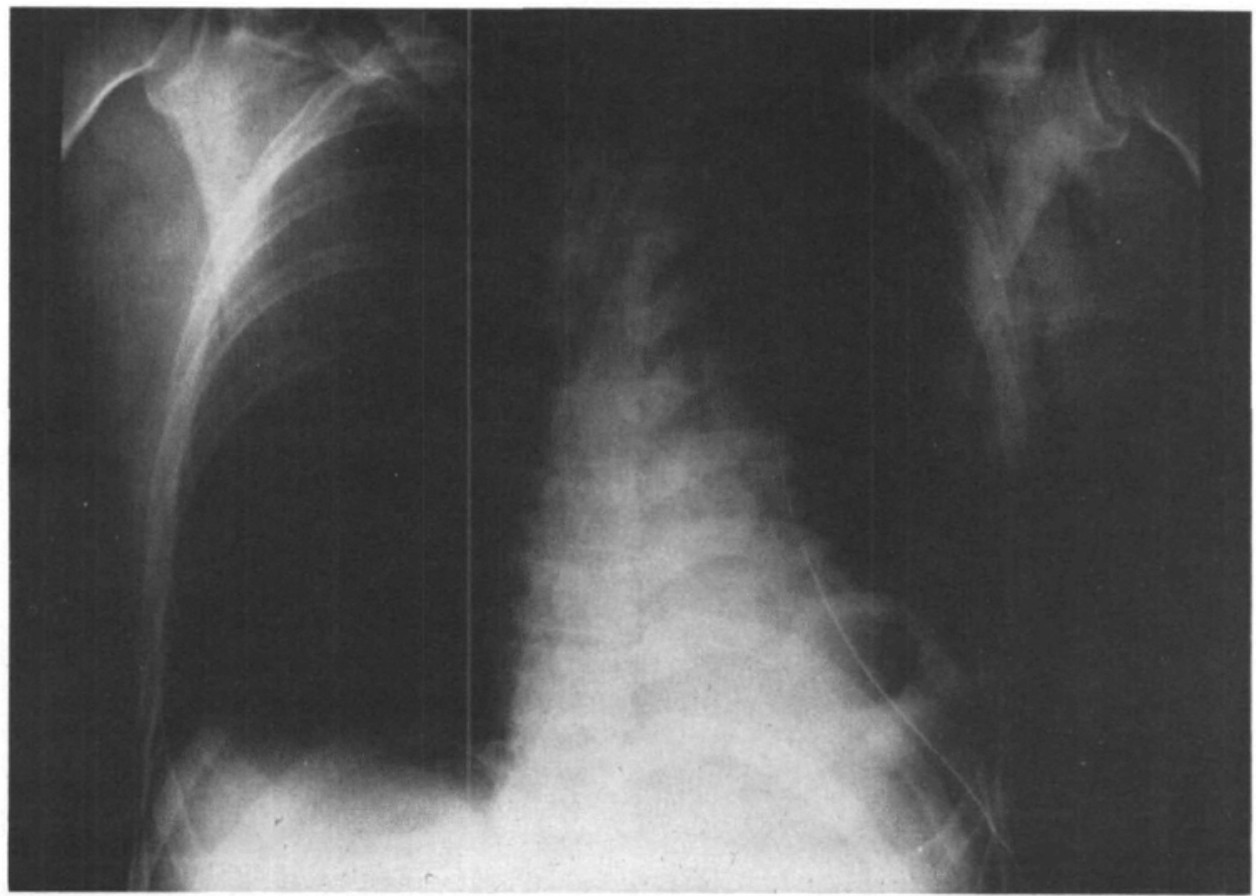

Figure 4. Case 3 (admission X-ray).

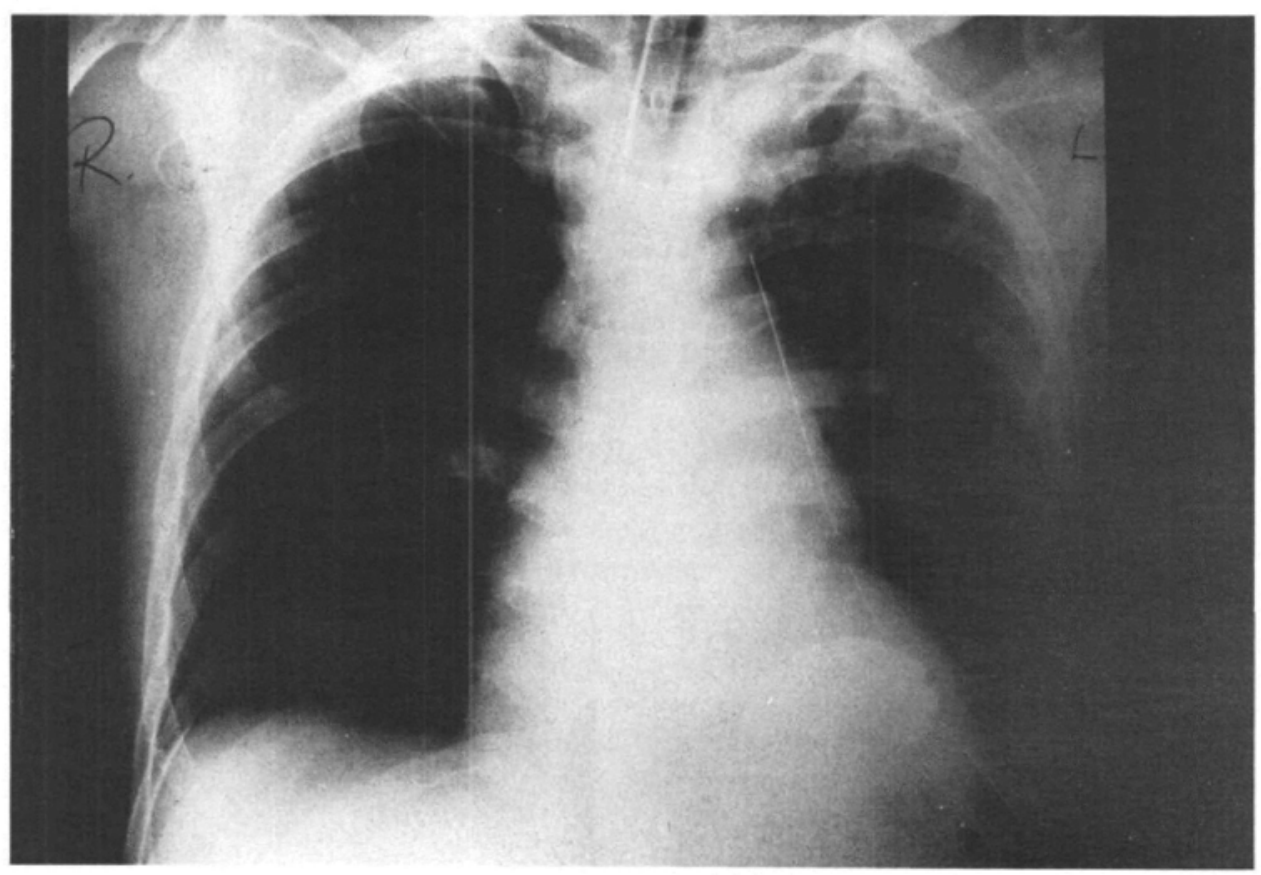

Ficure 5. Case 3 (after the introduction of barium contrast by a nasogastric tube). 


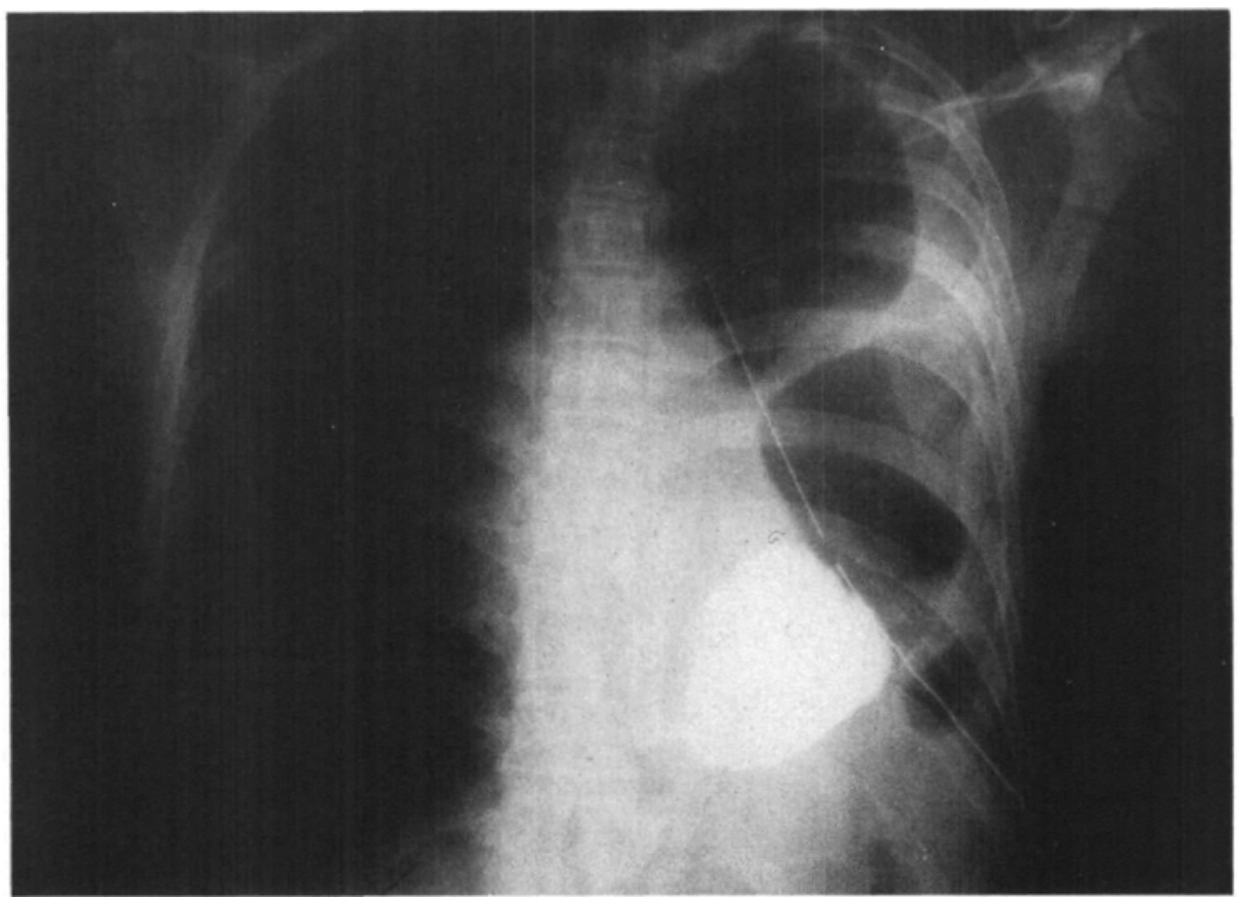

Figure 6. Case 3 (five days after the barium contrast was introduced).

hours. Subsequent recovery was greatly prolonged by recurrent pulmonary infections. At the time of discharge three months later, pulmonary function studies demonstrated a restrictive defect.

\section{Discussion}

All three patients presented with variable degrees of respiratory distress in association with other injuries, which prompted the taking of plain portable radiographs of the chest. This established the diagnosis of diaphragmatic hernia in the first two cases, and led to suspicion in the third. At no time were bowel sounds heard in the chest. Barium contrast proved to be of little value, since it was not the contrast itself but rather the subsequent development of an airdistended loop of bowel in the chest that established the diagnosis. Following confirmation of the diagnosis, surgical reduction of the hernia and its repair was the most immediate consideration.

One complication of this injury has been cardiovascular collapse on induction of anaesthesia using positive pressure ventilation. Loehning, Takaori and Safar ${ }^{5}$ reviewed three such cases, and after some further animal experimentation, postulated that the positive pressure had re-expanded lung previously compressed by the herniated abdominal contents. Since these organs were trapped in the involved hemithorax, any such re-expansion could only result in a shift of mediastinal structures to the opposite side. In turn, obstruction to the great veins from kinking often led to drastic falls in cardiac output. Such precipitating events in conjunction with hypoxia and acidosis were then thought to be responsible for these unexplained deaths. In conclusion, they recommended 
spontaneous ventilation after intubation with the patient awake until the hernia could be easily reduced. If assisted ventilation was necessary, then the use of low tidal volumes was considered preferable.

\section{Anaesthetic Management}

Once the diagnosis has been confirmed, an organized plan of anaesthesia and operation should be evolved with the above considerations kept in mind. The following is our suggested basic management:

Pre-operative preparation is mainly governed by the degree of respiratory distress and the consequent need for immediate surgical correction. Any time available should be used in cross-matching blood for transfusion and preparing the patient for the operation. As well as a large bore intravenous catheter in an arm, a medium-sized unit should be inserted into an external jugular vein for central venous pressure monitoring. The patient's condition is stabilized by blood volume replacement with crystalloid solutions, haemostasis, splinting, and oxygen supplementation. Tetanus toxoid is administered. A nasogastric tube should be inserted and suction applied to it. An indwelling urinary bladder catheter provides another valuable monitor of the shocked patient.

In the operating room, the patient is placed in the supine position with the shoulder of the involved side elevated by bolsters into a semi-lateral position giving good surgical access to either the chest or abdomen, since the incision may have to be extended into either cavity. The patient's skin is then prepared and draped while suitable monitoring equipment is positioned. With the surgeon ready to intervene, the trachea is intubated with the patient awake, using a lidocaine spray and titrated intravenous sedation as necessary. Where possible, spontaneous ventilation is allowed; but if assistance is necessary, low airway pressures and low tidal volumes are used. A sleep dose of thiopentone followed by nitrous oxide and oxygen is administered and this is followed by a narcotic just before the surgeon makes his incision. When decompression of the chest is imminent, a non-depolarizing muscle relaxant is given and ventilation is gently and gradually controlled. Once the abdominal contents have been removed from the chest, the lung is re-inflated by applying positive pressure. The anaesthetic is maintained in an appropriate manner, while the surgeons repair, excise, debride and obtain haemostasis.

Post-operatively, the patient is taken to an Intensive Care Unit with the tracheal tube in place for 24 to 48 hours of mechanical ventilation. In this manner, tracheal suctioning and humidification can be applied at a time when there is grave risk of pulmonary complications.

\section{Summary}

The aetiology and mechanism of traumatic diaphragmatic herniation have been presented. Three cases are described. Certain anaesthetic guidelines have been proposed to minimize possible complications inherent in this injury. This lesion may become much more frequent with the ever increasing number of motor vehicle accidents. 


\section{RÉSUMÉ}

La hernie diagphragmatique traumatique est une blessure que l'on peut rencontrer dans n'importe quel type d'accident impliquant un véhicule automobile. Elles se produisent probablement à la suite d'application d'un gradient de pression soudain au niveau du diaphragme ou encore par lapplication d'un mouvement de déchirement au niveau du diaphragme. Dans cette urgence chirurgicale, le grand danger consiste en la possibilité d'un collapsus cardiovasculaire à l'induction de l'anesthésie lorsque l'on utilise la ventilation à pression positive. L'application de la pression positive sur le poumon peut causer un déplacement aigu des structures médiastinales et une obstruction au retour veineux. Il est proposé pour éviter cette complication de procéder à une intubation de la trachée avant de procéder à l'induction et on procède à une ventilation contrôlée délicate seulement après que le chirurgien est prêt soit à entrer rapidement dans le thorax ou dans l'abdomen pour réduire rapidement la hernie. En post-opératoire, on ventile mécaniquement ces patients à cause des complications pulmonaires fréquentes associées à ce type de traumatisme.

\section{REFERENCES}

1. Andrus, C.H. \& Morton, J.H. Rupture of the diaphragm after blunt trauma. Amer. J. Surg. 119: 686 (1970).

2. Miller, J.D. \& HowiE, P.W. Traumatic rupture of the diaphragm after blunt injury. Brit. J. Surg. 55: 423 (1968).

3. Sterns, L.P., Jensen, N.K., Schmidt, W.R., Garamelra, J.J., \& Lynch, M.F. Diaphragmatic disruption in major thoracic trauma: a review of 16 cases. Canad. J. Surg. 12: 426 (1969).

4. SchWind, W.D. \& GALE, J.W. Late recognition and treatment of traumatic diaphragmatic hernias. Arch. Surg. 94: 330 (1967).

5. Loenning, R.W., Takaori, M., \& SafaR, P. Circulatory collapse from anaesthesia for diaphragmatic hernia. Arch. Surg. 90: 109 (1965). 